\title{
How should we best treat patients with bloodstream infections?
}

\author{
“The application of matrix-assisted laser desorption-ionization \\ time of flight mass spectrometry for the direct identification of \\ pathogens from positive blood culture, is among the major recent \\ revolutions in clinical microbiology laboratories."
}

Luis Eduardo López-Cortés' ${ }^{1}$ Marina de Cueto \& Jesús Rodríguez-Baño*,1

First draft submitted: 19 June 2017; Accepted for publication: 7 July 2017; Published online: 31 July 2017

Bloodstream infections (BSIs) are frequent and heterogeneous; they may occur in community and nosocomial infections from diverse sources in different types of patients, and are caused by different organisms. BSIs are also a major cause of morbidity and mortality in patients admitted to hospital. Therefore, management of patients with a BSI is a challenge, but the good news is that they are amenable to intervention.

BSIs must be pre-emptively managed globally, by promoting all preventive evidence-based measures (including hand hygiene, early withdrawal of unnecessary invasive devices, appropriate management of catheters, etc.). Once a BSI has occurred, adequate and early performance of blood cultures and rapid laboratory detection of the causative organism and its susceptibility are critical [1]. Blood culture remains the best approach to identify an etiological microorganism in a BSI but growing the bacteria or fungi can be time consuming. New diagnostic assays are now available for the detection and identification of bacteria and yeast in blood, providing earlier results than conventional subculture and phenotypic susceptibility testing [2]. However, many of the new technologies still require an initial growth of the pathogen in blood culture bottles and might not be rapid enough to be cost-effective and to have a substantial effect on clinical management. The application of matrix-assisted laser desorptionionization time of flight mass spectrometry for the direct identification of pathogens from positive blood culture, is among the major recent revolutions in clinical microbiology laboratories. Integration of matrix-assisted laser desorption-ionization time of flight into the laboratory workflow decreases the time to identify the organism by $1.2-1.5$ days compared with conventional methods; it may also be an alternative to rapidly detect methicillin-resistant Staphylococcus aureus and $\beta$-lactamaseproducing Enterobacteriaceae; therefore, a final report can be available to clinicians with a turnaround time of $<24 \mathrm{~h}$, allowing earlier appropriate treatment [3]. Another strategy is to apply rapid molecular

\section{KEYWORDS}

- antimicrobial therapy $\bullet$ bacteremia

- blood cultures $\bullet$ bloodstream infections $\bullet$ diagnostic tests $\bullet$ rapid microbiologic tests 


\author{
"A direct and fluid \\ communication between \\ clinical microbiologist \\ and infectious disease \\ specialist, ideally forming \\ a bacteremia team, is \\ imperative."
}

methods for the identification of pathogens in positive blood cultures. Peptide nucleic acid fluorescent in situ hybridization (PNA-FISH), QuickFISH, microarrays and PCR-based test allow the identification of species and specific resistance determinants for the most common organisms with a turnaround time of 2-4 h [4]. Probably, the greatest impact on patient outcomes may be achieved by the direct detection of pathogens in whole blood with multiplex or broad-range PCR assay. Its main advantage is the avoidance of the time-consuming culture step. However, like the conventional blood cultures, these assays also have their limitations; clinical specificity is challenged by the possibility of detecting nucleic acid of dead organisms, random nucleic acid that appears in blood transiently and reagent contamination from environmental organisms $[4,5]$.

The most important question is whether any rapid technique significantly affects physician behavior or improves patient care and outcomes. Traditionally, the most useful rapid test for blood cultures has been a Gram stain of the blood broth because it can be performed the moment a blood culture bottle is detected as positive and the result allows physicians to initiate or modify the antimicrobial therapy. The active notification of the Gram stain findings with written and oral reports and recommendations at bedside immediately after the detection of microbial growth in the blood culture has been shown to considerably increase the proportion of patients who received early appropriate treatment [6]; not less important is the clinical and economic benefits of close coordination between the microbiology laboratory and infectious disease specialist (IDS) for making decisions on treatment. In addition, EUCAST is developing guidance on performance of direct susceptibility testing on positive blood cultures (Kahlmeter G, Pers. Comm.). However, the impact of newer rapid methods for diagnosis of BSI on clinical and economic outcomes has been difficult to demonstrate. Overall, published data suggest that the use of rapid tests improved patients' outcomes when antibiotic stewardship programs assist clinicians in interpreting the results; however, in the absence of an antibiotic stewardship program, the clinical benefits of rapid methods may be reduced or absent $[7,8]$.

Once preliminary or definitive microbiological information is available, the selection of the best antimicrobial therapy and other aspects of early management and follow-up are far from easy. Specialized management of patients with BSI has been shown to improve quality of care and outcomes [9], and this is best done by quality improvement programs $[10,11]$. In fact, a positive blood culture provides an important opportunity for a potentially lifesaving intervention. Therefore, we do think that institutional comprehensive programs aimed at improving early reporting and specialized management of patient with BSI bacteremia, must be implemented in all centers [12]. A direct and fluid communication between clinical microbiologist and IDS, ideally forming a bacteremia team, is imperative.

The cornerstones of BSI management at the very moment the preliminary microbiological information is available are early administration of active antimicrobial therapy, the implementation of support measures in patients with sepsis and source control $[12,13]$. Regarding antimicrobial therapy, several aspects are to be taken into account including comorbidities (particularly, neutropenia, cancer, liver cirrhosis, renal insufficiency), risk factors for resistant bacteria (including previous microbiological results, previous antibiotic use, local rates), invasive procedures, source of the BSI, admission ward and criteria for sepsis or septic shock data [12]. Empirical regimen may be continued if considered appropriate or modified according to the available microbiological and clinical data.

Early identification of the source, which is many times not clearly apparent or confounding, is important as its appropriate management is critical (e.g., catheter withdrawal, abscess drainage, release of urinary or biliary tract obstructions, etc.) $[12,13]$. Also, the fact that blood cultures frequently yield only one pathogen, even in cases of typical polymicrobial infections, should be considered for therapeutic decisions, and obtaining samples from the source whenever possible is recommended.

Once the susceptibility results are available, antimicrobial therapy has to be reviewed again. The best antibiotic/s should be selected considering the identified microorganism/s and its minimum inhibitory concentrations, the pharmacokinetic/pharmacodynamic profiles of the drugs and the expected activity in the source of the bacteremia, their toxicity, interactions, spectrum and ecological impact [12]. In many cases, de-escalation to more specific, narrowerspectrum drugs can be recommended; while this is considered standard practice by many IDS, 
some physicians are reluctant to de-escalation. Therefore, we are now carrying out a multicenter randomized clinical trial in patients with BSI due to Enterobacteriaceae receiving empirical therapy with an antipseudomonal agent to demonstrate that de-escalation to a narrower targeted therapy is effective and safe in comparison to continuing with the empirical drug [14]. The dose should be carefully considered, taking the susceptibility of the microorganism, the renal and liver function, the source of infection and the clinical situation of the patient (hypotension, presence of third spaces, etc.) into account. Monotherapy is usually enough, with some exceptions (e.g., enterococcal endocarditis, severe Listeria monocytogenes infection, septic shock caused by carbapenemase-producing organisms, etc.).

Patients with BSI should be followed according to the features of the patient, the source infection and the microorganism. The objectives are to readily detect complications and recurrences, and provide an adequate duration of therapy, with switch to oral drugs if possible. This must be done by the bacteremia team and has to be structured and planned from the first visit. Some examples of follow-up activities include performance of blood cultures after 48-72 h of active therapy which should be done in all cases of BSI due to Staphylococcus aureus, Candida spp. and possibly Enterococcus spp., as well as in all patients with predisposing factors for endocarditis; echocardiography in selected patients; or fundoscopy in candidemia [12,15-17]. Quality indicators for management have been developed for $S$. aureus, candidemia and catheter-associated BSI [12,15,17-18]. Experience with switching to oral therapy is increasing and overall seems safe if the following conditions are fulfilled: availability of an active oral drug with adequate bioavailability, clinical stability and adequate control of the source/s of BSI is achieved, adequate oral intake, and absence of gastrointestinal conditions that might jeopardize drug absorption. A randomized trial is being performed to evaluate the efficacy and safety or early switch to oral therapy in $S$. aureus bacteremia [19].

In summary, the management of BSI is complex, several aspects of BSI management are associated with the outcome of patients, and therefore amenable of intervention. This should be performed by a multidisciplinary specialized team. The diagnostic tests to be used need to be elected at each institution, but we encourage the use of rapid diagnostic tests in addition to conventional methods for blood cultures as part of a bacteremia program, including early interventions and follow-up to improve clinical outcomes.

\section{Financial \& competing interests disclosure}

The authors received support for research by Plan Nacional de I+D+i 2013-2016 and Instituto de Salud Carlos III, Subdirección General de Redes y Centros de Investigación Cooperativa, Ministerio de Economía, Industria y Competitividad, Spanish Network for Research in Infectious Diseases (REIPI RD16/0016/0001) - co-financed by European Development Regional Fund ERDF 'A way to achieve Europe,' Operative Program Intelligent Growth 2014-2020. J Rodríguez-Baño received honoraria for collaborating in the design and coordination of a research project from AstraZeneca and for participating in accredited educational activities from Merck. The authors have no other relevant affliations or financial involvement with any organization or entity with a financial interest in or financial conflict with the subject matter or materials discussed in the manuscript apart from those disclosed.

No writing assistance was utilized in the production of this manuscript.

\section{References}

1 Rhodes A, Evans LE, Alhazzani W et al. Surviving Sepsis Campaign: international guidelines for management of sepsis and septic shock: 2016. Crit. Care Med. 45(3), 486-552 (2017).

2 Opota O, Croxatto A, Prod'hom Get al. Blood culture-based diagnosis of bacteraemia: state of the art. Clin. Microbiol. Infect. 21(4), 313-322 (2015).

3 Stevenson LG, Drake SK, Murray PR. Rapid identification of bacteria in positive blood culture broths by matrix-assisted laser desorption ionization-time of flight mass spectrometry. J. Clin. Microbiol. 48(2), 444-447 (2010).

4 Peters RP, van Agtmael MA, Danner SA et al. New developments in the diagnosis of bloodstream infections. Lancet Infect. Dis. 4(12), 751-760 (2004).

5 Opota O, Jaton K, Greub G. Microbial diagnosis of bloodstream infection: towards molecular diagnosis directly from blood. Clin. Microbiol. Infect. 21(4), 323-331 (2015).

6
Bouza E, Sousa D, Muñoz P et al. Bloodstream infections: a trial of the impact of different methods of reporting positive blood culture results. Clin. Infect. Dis. 39(8),1161-1169 (2004).

7 Buehler SS, Madison B, Snyder SR et al. Effectiveness of practices to increase timeliness of providing targeted therapy for inpatients with bloodstream infections: a laboratory medicine best practices systematic review and meta-analysis. Clin. Microbiol. Rev. 29(1), 59-103 (2016).

8 Huang AM, Newton D, Kunapuli A et al. Impact of rapid organism identification via matrix-assisted laser desorption/ionization time-of-flight combined with antimicrobial 
stewardship team intervention in adult patients with bacteremia and candidemia. Clin. Infect. Dis. 57(9), 1237-1245 (2013).

9 Byl B, Clevenbergh P, Jacobs F et al. Impact of infectious diseases specialists and microbiological data on the appropriateness of antimicrobial therapy for bacteremia. Clin. Infect. Dis. 29(1), 60-66 (1999).

10 Minton J, Clayton J, Sandoe J et al. Improving early management of bloodstream infection: a quality improvement project. BMJ 336(7641), 440-443 (2008).

11 López-Cortés LE, Del Toro MD, GálvezAcebal J et al. Impact of an evidence-based bundle intervention in the quality-of-care management and outcome of Staphylococcus aureus bacteremia. Clin. Infect. Dis. 57(9), 1225-1233 (2013).

12 Rodríguez-Baño J, de Cueto M, Retamar P et al. Current management of bloodstream infections. Expert Rev. Anti. Infect. Ther. 8(7), 815-829 (2010).
13 Dellinger RP, Levy MM, Rhodes A et al. Surviving Sepsis Campaign: international guidelines for management of severe sepsis and septic shock: 2012. Intensive Care Med. 39(2), 165-228 (2013).

14 López-Cortés LE, Rosso-Fernández C, Núñez-Núñez M et al. Targeted simplification versus antipseudomonal broad-spectrum betalactams in patients with bloodstream infections due to Enterobacteriaceae (SIMPLIFY): a study protocol for a multicentre, open label, Phase III randomised, controlled, non-inferiority clinical trial. $B M J$ Open doi:10.1136/bmjopen-2016-015439 (2017) (Epub ahead of print)

15 Pappas PG, Kauffman CA, Andes DR et al. Clinical practice guideline for the management of candidiasis: 2016 update by the Infectious Diseases Society of America. Clin. Infect. Dis. 62(4), e1-e50 (2016).

16 Bai AD, Agarwal A, Steinberg M et al. Clinical predictors and clinical prediction rules to estimate initial patient risk for infective endocarditis in Staphylococcus aureus bacteremia: a systematic review and metaanalysis. Clin. Microbiol. Infect. doi:10.1016/j. cmi.2017.04.025 (2017) (Epub ahead of print).

17 Bouza E, Kestler M, Beca T et al. The NOVA score: a proposal to reduce the need for transesophageal echocardiography in patients with enterococcal bacteremia. Clin. Infect. Dis. 60(4), 528-535 (2015).

18 Mermel LA, Allon M, Bouza E et al. Clinical practice guidelines for the diagnosis and management of intravascular catheter-related infection: 2009 update by the Infectious Diseases Society of America. Clin. Infect. Dis. 49(1), 1-45 (2009).

19 Kaasch AJ, Fätkenheuer G, PrinzLangenohl R et al. Early oral switch therapy in low-risk Staphylococcus aureus bloodstream infection (SABATO): study protocol for a randomized controlled trial. Trials 16, 450 (2015) 